\title{
Multifractal analysis of vertical profiles of soil penetration resistance at the field scale
}

\author{
G. M. Siqueira ${ }^{1}$, E. F. F. Silva ${ }^{1}$, A. A. A. Montenegro ${ }^{1}$, E. Vidal Vázquez ${ }^{2}$, and J. Paz-Ferreiro ${ }^{2,3}$ \\ ${ }^{1}$ Department of Rural Technology, Federal Rural University of Pernambuco, Dom Manoel de Medeiros s/n, \\ 52171-900 Recife-PE, Brazil \\ ${ }^{2}$ Faculty of Sciences, University of Coruña, Campus A Zapateira, 15008 Coruña, Spain \\ ${ }^{3}$ Department of Edaphology, Polytechnic University of Madrid, Av. Complutense sn, 28040 Madrid, Spain
}

Correspondence to: J. Paz-Ferreiro (jpaz@udc.es)

Received: 31 August 2012 - Revised: 6 May 2013 - Accepted: 9 June 2013 - Published: 23 July 2013

\begin{abstract}
Soil penetration resistance (PR) is widely used as an indirect indicator of soil strength. Soil PR is linked to basic soil properties and correlated to root growth and plant production, and as such it is extensively used as a practical tool for assessing soil compaction and to evaluate the effects of soil management. This study investigates how results from multifractal analysis can quantify key elements of depth-dependent soil PR profiles and how this information can be used at the field scale. We analysed multifractality of $50 \mathrm{PR}$ vertical profiles, measured from 0 to $60 \mathrm{~cm}$ depth and randomly located on a 6.5 ha sugar cane field in northeastern Brazil. The scaling property of each profile was typified by singularity, and Rényi spectra estimated by the method of moments. The Hurst exponent was used to parameterize the autocorrelation of the vertical PR data sets. The singularity and Rènyi spectra showed that the vertical PR data sets exhibited a well-defined multifractal structure. Hurst exponent values were close to 1 , ranging from 0.944 to 0.988 , indicating strong persistence in PR variation with soil depth. Also, the Hurst exponent was negatively and significantly correlated to coefficient of variation (CV), skewness and maximum values of the depth-dependent PR. Multifractal analysis added valuable information to describe the spatial arrangement of depth-dependent penetrometer data sets, which was not taken into account by classical statistical indices. Multifractal parameters were mapped over the experimental field and compared with mean and maximum values of PR. Combination of spatial variability survey and multifractal analysis appear to be useful to manage soil compaction.
\end{abstract}

\section{Introduction}

Soil strength is defined as the resistance that has to be overcome to cause soil physical deformation. Soil strength is an important soil physical property as it affects basic aspects of agricultural soils, including root growth, seedling emergency, compaction, erodibility, trafficability and bearing capacity (Guérif, 1990; Soane and Van Ouwerkerk, 1994). Direct measurement of soil strength properties are difficult to perform, so they have remained until now rather scarce. Instead, most frequently, soil resistance to deformation is indirectly and empirically assessed by measuring the resistance to penetration of a metallic plunger with a particular shape, usually a cone, which is referred to as a penetrometer (e.g. ASAE, 1986). Thus, penetrometers are simple and relatively inexpensive devices, easily providing an indirect indication of soil strength.

Penetration resistance is an easily measurable parameter which has been found to depend on basic soil properties that are vital for plant growth, mainly soil composition, structure, bulk density and water content. In addition, PR correlates with several properties of agronomic importance, such as crusting, compaction or vehicle trafficability. Thus, PR has been often used as a surrogate measurement of key soil properties (e.g. Dexter et al., 2007). Indeed, penetrometers have been utilized in a number of field experiments to assess soil compaction (e.g. O'Sullivan et al., 1987) or the effects of soil management (e.g. Castrignanó et al., 2002). Conversely, equations for predicting penetrometer resistance from soil properties have been proposed (e.g. Dexter et al., 2007; Vaz et al., 2011). Moreover, soil PR above a given threshold has 
been shown to affect root penetration and hence crop production (Häkansson et al., 1988). Consequently, field penetrometers also have been frequently employed for estimating resistance to root growth in soil as soil PR is a rough indicator of the pressure encountered by roots for elongation.

Nowadays, micropenetrometers are increasingly used in laboratory conditions. This is because of the wide acceptance of a quality index based on three main soil physical properties: soil strength, matric potential and aeration. This index was defined by Letey (1985) as "the range of moisture content at which plant water uptake is neither limited by soil resistance when dry, nor by poor aeration when too wet", and is now referred to as "least limiting water range" (LLWR), following Da Silva et al. (1994). Soil strength data required for LLWR evaluation are readily provided by PR measurements.

Soils are highly variable and heterogeneous over several spatial scales. Over the past few decades scaling analysis, such as fractal and multifractal analysis have been applied to characterize soil properties and processes, and many studies have revealed scale-dependent patterns of soil variability from very short distances (e.g. Pachepsky et al., 1996; PazFerreiro et al., 2010) to geomorphologic landscapes units (Biswas et al., 2012), or even continents and the whole planetary scale (Caniego et al., 2006). Both fractal and multifractal scaling assume a hierarchical distribution of mass in space so that the whole results from the union of similar subsets. While in the fractal approach subsets are considered similar to the whole, multifractal distributions hypothesize that subsets are related to the whole through varying scaling factors (e.g. Tarquis et al., 2003). As a consequence, in monofractal scaling, one single exponent is sufficient to characterize the scaling behaviour, whereas multifractal scaling involves a continuous spectrum of scale exponents, which are related to different intensity levels of complex processes (Everstz and Mandelbrot, 1992; Falconer, 1997).

Fractal geometry showed ability to address the complex relations between a range of soil properties and soil-forming factors and processes that do not operate disconnectedly but as an ensemble of interacting forces driving complex and nested effects, intuitively related to fractal or multifractal behaviour. For example, heterogeneity, tortuosity and connectivity of the soil pore/solid space have been quantified using fractal (e.g. Pachespsky et al., 1996) or multifractal techniques (e.g. Tarquis et al., 2003; Paz-Ferreiro et al., 2010; San José Martínez et al., 2010). Likewise, soil surface microrelief has been described using fractal models (e.g. Huang and Bradford, 1992; Vidal Vázquez et al., 2005, 2007) and multifractal models (e.g. Vidal Vázquez et al., 2008; García Moreno et al., 2010).

Early attempts to model the spatial variation of soil properties using fractal theory rely on the fractional Brownian motion, i.e. a $f \mathrm{Bm}$ model (Burrough, 1983; Armstrong, 1986). A $f \mathrm{Bm}$ is an expansion of the random walk or Brownian motion model $(\mathrm{Bm})$ which is characterized by the Hurst exponent $H=0.5$, and was first proposed by Mandelbrot and van
Ness (1968). The Bm model corresponds to a Gauss-Markov process, i.e. a random process consisting of a sequence of discrete steps of fixed length, where correlation between successive values vanishes. For a $f \mathrm{Bm}$ model the Hurst parameter $H,(0<H<1)$, is directly related to the long-term memory of the studied variable. The range $1 / 2<H<1$ is usually associated with persistence (positive autocorrelation) or long-range dependencies, whereas the range $0<H<1 / 2$ represents anti-persistent behaviour (negative autocorrelation) and short-range dependencies (e.g. Feder, 1988). More recently, it has been also recognized that a large class of multifractal processes could be approximated by the multifractal Brownian motion $(\mathrm{mBm})$ models, based on the fact that the power law behaviour of second-order statistics allows estimation of a generalized Hurst scaling function (e.g. Leland et al., 1994; San José Martínez et al., 2010). It is worth nothing that, similar to the Hurst exponent, the generalized Hurst scaling function can be categorized into three types, i.e. persistent, entirely random distribution and anti-persistent.

Several studies also have reported self-affine scaledependent patterns and singularity of the spatial variation of soil PR itself in horizontal layers (e.g. Usowicz and Lipiec, 2009; Pérez et al., 2010). Furthermore, multifractal analysis has been used to assess patterns of spatial variation of penetrometric data sets measured along transects (Folorunso et al., 1994) or on planes (Roisin, 2007), providing valuable information to better understand the inner structure of PR for horizontal soil layers. However, to the best of our knowledge, until now multifractal analysis has not been applied to characterize the spatial heterogeneity of depth-dependent, vertical resistance profiles obtained by penetrometer. Therefore, the objectives of this work were (a) to characterize the intrinsic variability and scaling of PR vertical profiles using multifractal models and (b) to assess compaction at the field scale by mapping classical descriptive statistics and multifractal parameters.

\section{Material and methods}

\subsection{Description of the experimental site}

The field experiment was conducted at an agricultural research site located in Goiania municipality, Pernambuco State, northeast Brazil (latitude $07^{\circ} 34^{\prime} 25^{\prime \prime} \mathrm{S}$, longitude $34^{\circ} 55^{\prime} 39^{\prime \prime} \mathrm{W}$ ) (Fig. 1a). The studied field, located $10 \mathrm{~km}$ inland from the Atlantic Ocean, is 6.5 ha in surface, and has a mean altitude of $8.5 \mathrm{~m}$ a.s.l. This field is representative of a regional lowlands landscape, whose soils are affected by seawater salinity, with plantations of sugarcane (Saccharum officinarum sp.) as the main economic activity. Sugarcane has been cropped as a monoculture during the last $24 \mathrm{yr}$, and it was managed by burning the straw each year after harvesting. The plantation was renewed every seven years. 


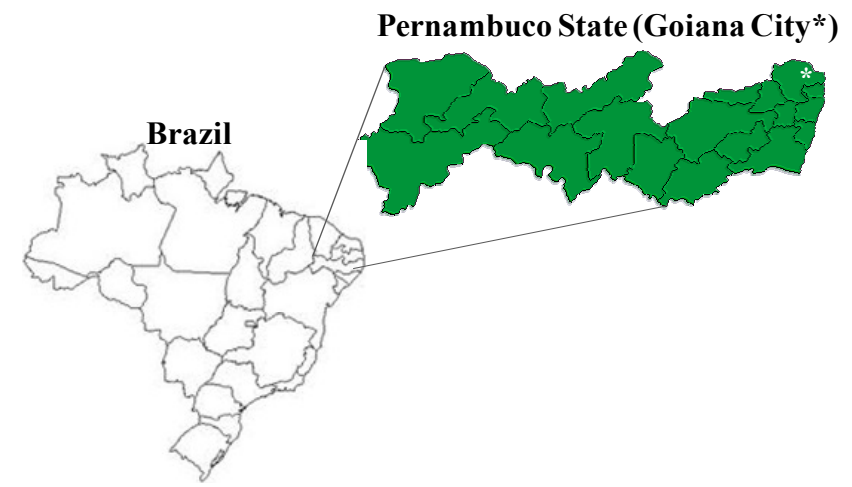

Fig. 1a. Location of the experimental site.

According to Köppen the local climate, characterized by constant high temperatures and a rainfall regime with contrasting wet and a dry seasons, was defined as tropical (As type, also referred to as "pseudo-tropical"). Mean annual temperature is about $24^{\circ} \mathrm{C}$ and mean yearly precipitation reaches approximately $1800 \mathrm{~mm}$, from which about $1250 \mathrm{~mm}$ correspond to the rainy season, lasting from October to March during austral summer.

Soil was classified as an Orthic Podzol (Soil Survey Staff, 2010), which is equivalent to an "Espodossolo Humilúvico órtico" following the Brazilian Soil Classification System (EMBRAPA, 2006). Soil parent material consists of highly weathered sediments from continental origin, dating from the late Tertiary and belonging to the Barreiras group (Brasil, 1969, 1972).

Soil properties were analysed following methods described in Camargo et al. (1986). As shown in Table 1, the soil exhibited a sandy texture with a sand content of about $930 \mathrm{~g} \mathrm{~kg}^{-1}$ and the bulk density increased with depth from $1.52 \mathrm{~kg} \mathrm{dm}^{-3}$ at the topsoil $(0-20 \mathrm{~cm})$ to $1.66 \mathrm{~kg} \mathrm{dm}^{-3}$ at $>1.00 \mathrm{~m}$ depth.

\subsection{Penetrometer resistance data set}

Soil penetration resistance data were collected at 50 points randomly located across the experimental field (Fig. 1b). Penetrometer measurements were done on 27 January 2012. Cumulative rainfall in the period from 9 to 25 January was $386.5 \mathrm{~mm}$. Even though on 27 January $0.5 \mathrm{~mm}$ rain was recorded, this figure can be considered as negligible because of the high evaporation. Thus, data collection was performed two days after important rainfall had ceased, which corresponds to the field capacity condition. Mean soil water content increased with depth from $34.5 \mathrm{~m}^{3} \mathrm{~m}^{-3}$ at $0.0-0.3 \mathrm{~m}$ depth to $47.2 \mathrm{~m}^{3} \mathrm{~m}^{-3}$ at depths higher than $1 \mathrm{~m}$ (Table 1), showing the effect of the subsurface water table.

Penetration resistance was measured with a dynamic hammer penetrometer (model IAA/Planalsucar; Stolf, 1991) at all the points marked over the field. This meant the vertical stress of a steel cone with an angle of $30^{\circ}$ and basal diame-
Table 1. Texture, bulk density and water content at the sampling date.

\begin{tabular}{cccccc}
\hline \multirow{2}{*}{$\begin{array}{c}\text { Depth } \\
(\mathrm{m})\end{array}$} & \multicolumn{2}{c}{$\begin{array}{c}\text { Texture } \\
\left(\mathrm{g} \mathrm{kg}^{-1}\right)\end{array}$} & \multirow{2}{*}{$\begin{array}{c}\text { Bulk density } \\
\left(\mathrm{kg} \mathrm{dm}^{-3}\right)\end{array}$} & $\begin{array}{c}\text { Water content } \\
\left(\mathrm{m}^{3} \mathrm{~m}^{-3}\right)\end{array}$ \\
\cline { 2 - 4 } & clay & silt & sand & & \\
\hline $0.0-0.3$ & 44 & 26 & 930 & 1.52 & 34.50 \\
$0.3-0.6$ & 43 & 25 & 932 & 1.54 & 36.80 \\
$0.6-1$ & 44 & 26 & 930 & 1.60 & 42.60 \\
$>1$ & 32 & 40 & 928 & 1.66 & 47.20 \\
\hline
\end{tabular}

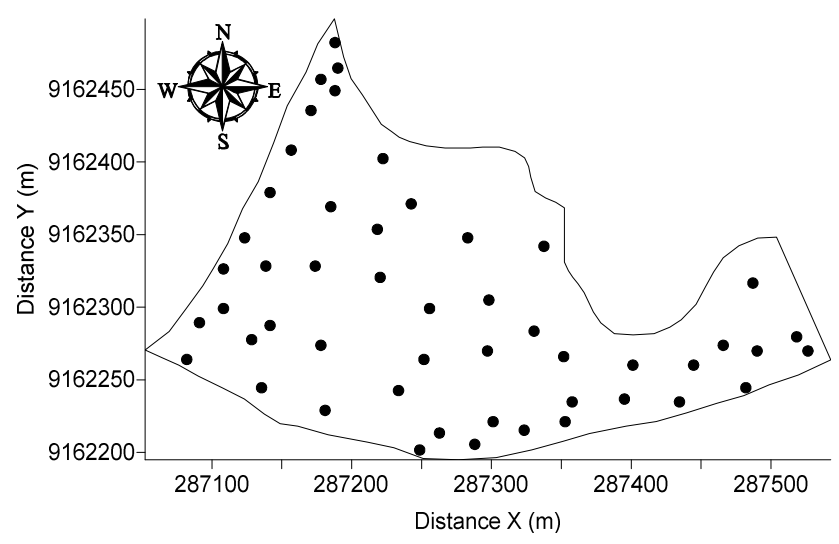

Fig. 1b. Sampling scheme for penetration resistance measurements.

ter of $1.25 \mathrm{~cm}$ (ASAE, 1986) to $60 \mathrm{~cm}$ depth was manually determined. At each point, PR data sets were obtained at intervals of $1 \mathrm{~cm}$ as described by Stolf (1991) and expressed in MPa units.

\subsection{Multifractal analysis and generalized Hurst exponent}

Multifractal analysis was implemented following the moment method. In addition, the traditional Hurst exponent, originally developed for monofractal series, has been generalized and used to parameterize scaling heterogeneity (multifractality).

To implement multifractal analysis, suppose $\mu$ (S) represents a measure (field) defined on a set $\mathrm{S}$ associated with a geometrical support. To assess the heterogeneity of the measure, $\mu_{i}$, the space $R^{D}$ can be split into $n$ boxes of the same linear size $\delta$ (equal intervals in $R^{1}$ ) by sequential divisions (e.g. Evertsz and Mandelbrot, 1992; Caniego et al., 2006). Considering a one-dimensional profile of length $L$, this involves successive partitioning into $k$ stages $(k=1,2,3 \ldots)$. Hence, at each scale, $\delta$, a number of segments, $N(\delta)=2^{k}$, are obtained with characteristic size length, $\delta=L \times 2^{-k}$, covering the whole extent of the support, $L$. In this study the interval of depths $[1,60]$ was taken as the support (in $\mathrm{cm}$ ). Thus, the total profile depth was subdivided into subintervals 
of equal length, $\delta$ (e.g. Miranda et al., 2006; San José Martínez et al., 2010), obtained with $1 \leq k \leq 5$.

Then, the distribution of the studied data series was normalized by introducing a new variable, the probability mass function, $\mu_{i}(\delta)$. This variable describes the portion of total mass contained in each segment, and was estimated as

$\mu_{i}(\delta)=N_{i}(\delta) / N_{\mathrm{t}}$,

where $N_{i}(\delta)$ is the value of the measure in the $i$-th segment of size $\delta$, and $N_{\mathrm{t}}$ represents the total mass in the whole transect.

For each box or segment, the probability mass function, $\mu_{i}(\delta)$, scales with the box or interval size, $\delta$, as

$\mu_{i}(\delta) \propto \delta^{\alpha_{i}}$,

where $\alpha_{i}$ is the singularity or Lipschitz-Hölder or Hölder exponent characterizing density in the $i$-th box (Halsey et al., 1986). The Hölder exponent, given by $\alpha_{i}=\log \mu_{i}(\delta) / \log \delta$, may be interpreted as a crowding index for the degree of concentration of the measure, $\mu$. It is, in fact, the logarithmic density of the $i$-th box of the partition of characteristic size $\delta$.

Also, for multifractal distributed measures, the number $N_{\delta}(\alpha)$ of cells of size $\delta$, having a singularity or Hölder exponent equal to $\alpha$, increases for decreasing $\delta$ and obeys a power law:

$N(\alpha) \propto \delta^{-f(\alpha)}$,

where the exponent $f(\alpha)$ is a continuous function of $\alpha$ denoted as singularity spectrum.

Based on the scaling properties of the normalized variable $\mu_{i}(\delta)$ different scaling functions can be estimated using either the moment or the direct method. The box-countingbased moment method (Evertsz and Mandelbrot, 1992) relies on the so-called partition function defined as

$\chi(q, \delta)=\sum_{i=1}^{n(\delta)} \mu_{i}^{q}(\delta)$,

where $q$ is a real number $(-\infty<q<-\infty)$ and $n(\delta)$ is the number of cells of size $\delta$.

A $\log -\log$ plot of the quantity $\chi(q, \delta)$ versus $\delta$ for different values of $q$ yields

$\chi(q, \delta) \propto \delta^{-\tau(q)}$,

where $\tau_{q}$ is the mass scaling function of order $q$. The method of moments is only justified if the plots of $\chi(q, \delta)$ versus $\delta$ are straight lines (Hasley et al., 1986). The mass exponent function $\tau_{q}$ was estimated from the partition function with Eq. (1) (Table 2). The function $\tau_{q}$ controls how the moment of measure $\mu_{i}$ scales with $q$. In general, multifractal measures yield a nonlinear function of $\tau_{q}$, whereas a monofractal corresponds to linear $\tau_{q}$.
Table 2. Algorithms used for computing mass exponent function, $\tau_{q}$, singularity spectrum $f(\alpha)$ versus $\alpha_{q}$ and generalized dimension, $D_{q}$, by the moment method.

$$
\begin{aligned}
& \text { Mass exponent function, } \tau_{q} \\
& \tau(q)=\lim _{\delta \rightarrow 0} \frac{\log \chi(q, \delta)}{\log (1 / \delta)}
\end{aligned}
$$

Singularity strength, $\alpha_{q}$, and multifractal spectrum, $f(\alpha)$

$$
\alpha(q) \propto \frac{\sum_{i=1}^{N(\delta)} \mu_{i}(q, \delta) \log \left[\mu_{i}(\delta)\right]}{\log (\delta)}
$$

$$
f(\alpha(q)) \propto \frac{\sum_{i=1}^{N(\delta)} \mu_{i}(q, \delta) \log \left[\mu_{i}(q, \delta)\right]}{\log (\delta)}
$$

$$
\begin{aligned}
& \text { Generalized dimension, } D_{q} \\
& D_{q}=\tau(q) /(q-1) \\
& D_{q}=\lim _{\delta \rightarrow 0} \frac{1}{q-1} \frac{\log [\chi(q, \delta)]}{\log \delta} \\
& D_{1}=\lim _{\delta \rightarrow 0} \frac{\sum_{i=1}^{n(\delta)} \chi_{i}(1, \delta) \log \left[\chi_{i}(1, \delta)\right]}{\log \delta}
\end{aligned}
$$

The scaling functions denoted as singularity spectrum, $f(\alpha)$, and local scaling index, $\alpha_{q}$, also might be estimated from the mass exponent function, $\tau_{q}$, through a Legendre transformation. However, in this work these functions have been obtained by the direct method (Chhabra and Jensen, 1989). This method relies on the quantities $\mu_{i}(q, \delta)$ that correspond to contributions of individual segments of the partition function, defined as

$\mu_{i}(q, \delta)=\mu_{i}^{q}(\delta) / \sum_{1}^{n(\delta)} \mu_{i}^{q}(\delta)$

Then, using a set of real numbers, $-\infty<q<\infty$, the functions $\alpha_{q}$ and $f(\alpha)_{q}$ can be estimated by Eqs. (2a) and (2b), respectively, presented in Table 2 .

The graph of $f(\alpha)$ versus $\alpha$, called the multifractal spectrum, typically has a parabolic concave downward shape, with the range of $\alpha$ values increasing with the increase in the heterogeneity of the measure. The minimum scaling exponent $\left(\alpha_{q+}\right)$ corresponds to the most concentrated region of the measure, whereas the maximum exponent $\left(\alpha_{q-}\right)$ corresponds to the rarefied regions of the measure. 
Additionally, the scaling functions denoted as mass exponent function, $\tau_{q}$, and generalized dimension, $D_{q}$, are also related (Hentschel and Procaccia, 1983), as defined by Eq. (3a) (Table 2). In fact, the concept of generalized dimension, $D_{q}$, corresponds to the scaling exponent for the $q$-th moment of the measure. Based on the work of Rényi (1955) generalized dimensions can be also defined by Eq. (3b) (Table 2). However, using Eqs. (3a) or (3b), $D_{1}$ becomes indeterminate because the value of the denominator is zero. Therefore, for the particular case that $q=1$, an equivalent equation is obtained, using L'Hôpital's rule (Eq. 3c, Table 2).

For a monofractal, $D_{q}$ is a constant function of $q$; thus no additional information is obtained by examining higher moments. However, for multifractal measures, the relationship between $D_{q}$ and $q$ is not constant. In this case, the most frequently used generalized dimensions are $D_{0}$ for $q=0, D_{1}$ for $q=1$ and $D_{2}$ for $q=2$, which are referred to as capacity, information (Shannon entropy) and correlation dimension, respectively.

The capacity or box-counting dimension, $D_{0}$, is independent of the quantity of mass in each box; it is the scaling exponent of the number of non-empty boxes and takes into account the fact that the boxes are occupied or not. The information dimension, $D_{1}$, gives the probability of occupation of the $i$-th box of size $\delta$, without taking into account the way in which the measure is distributed within each box. Thus, $D_{1}$ provides a physical characterization, indicating how heterogeneity changes across a certain range of scales, and it is also related to the Shannon entropy index (Grassberger, 1983). The correlation dimension, $D_{2}$, describes the uniformity of the measure values among intervals. The generalized dimension, $D_{q}$, may be more useful for the comprehensive study of multifractals. Differences between $D_{q}$ allow comparison of the complexity of the measure. In homogeneous structures, $D_{q}$ is close, whereas in a monofractal they are equal.

Now we consider a stationary stochastic process defined by $X=\left(X_{i}: i=0,1,2, \ldots\right)$, with constant mean $\mu$ and finite variance $\sigma^{2}$. The autocorrelation function at lag $k, \rho(k)$, of a stochastic data series is defined as

$\rho(k)=E\left[\left(X_{i}-\mu\right)\left(X_{i+k}-\mu\right)\right] / E\left[\left(X_{i}-\mu\right)^{2}\right]$,

where $E$ stands for the mathematical expectation and depends only on $k$. Moreover, if the series, $X_{i}$, has a longrange dependency, the autocorrelation function, $\rho(k)$, scales following the power law

$\rho(k) \propto k^{h(q)}$,

where $h(q)$ is the generalized Hurst scaling function. For simplicity, next will be assume that the stochastic process of interest is a second-order stationary process (i.e. a zero mean and first and second moments that do not change over position). In this particular case the Hurst exponent measures the power law behaviour of the autocorrelation function: $\rho(k) \propto k^{2 H-2}$ (Leland et al., 1994; San José Martínez et al., 2010). Thus, the scaling exponent provides information about long-term memory of the spatial or temporal series of interest.

On the other hand, the correlation dimension, $D_{2}$, appraises the average fluctuation of the spatial series. Hence, both $D_{2}$ and the Hurst exponent $H$ account for the scaling property of second-order stationary data sets. Therefore, as proposed by Riedi et al. (1999) in multifractal stochastic processes the Hurst exponent is related to the correlation dimension by

$D_{2}=2 H-1$

Consequently, multifractal analysis also allows parameterization of the log-range dependencies by the Hurst exponent, $H$.

\subsection{Statistical analysis and spatial representation over the experimental field}

Preliminary statistical analysis - involving calculation of mean, variance, standard deviation, coefficient of variation (CV), skewness and kurtosis - was performed using the STAT code described in Vieira et al. (2002). Correlation analyses between statistical parameters and multifractal indices were made by Spearman ranking using the SAS package, version 8.0 (SAS Institute, 1999).

Both PR variables and multifractal indices evaluated for the 50 positions marked over the experimental field were subjected to semivariogram analysis (e.g. Paz et al., 1996; Vieira et al., 2002). Nevertheless, plots of semivariance versus lag distance were nearly flat, and as such overall the pattern of spatial dependence was described by the pure nugget effect model. The lack of spatial dependence prevented the kriging to be used for mapping purposes. Therefore, maps to visualize spatial distribution of PR, statistical and multifractal indices were made by means of the inverse distance method, described elsewhere (e.g. Burrough and Mc Donnell, 1998; Caridad-Cancela et al., 2005).

\section{Results and discussion}

\subsection{Statistical analysis of penetration resistance}

Mean and standard deviation of the PR measured over the 50 positions from surface $0 \mathrm{~cm}$ to $60 \mathrm{~cm}$ depth are shown in Fig. 2. On average, PR over the entire field increased from $0 \mathrm{MPa}$ at the surface to $2 \mathrm{MPa}$ at about $40 \mathrm{~cm}$ depth. Because standard deviation also increased with depth, CVs of the mean PR involved rather uniform, moderate values (below $40 \%$ ) for the successive depths, except for the first $7 \mathrm{~cm}$ near the soil surface, which exhibited higher CV values. Soil composition (i.e. texture, organic matter content), water content and bulk density largely influence soil PR (e.g. Da Silva et al., 1994; Dexter et al., 2007; Vaz et al., 2011). Soil samples collected at successive depths were coarse textured and 


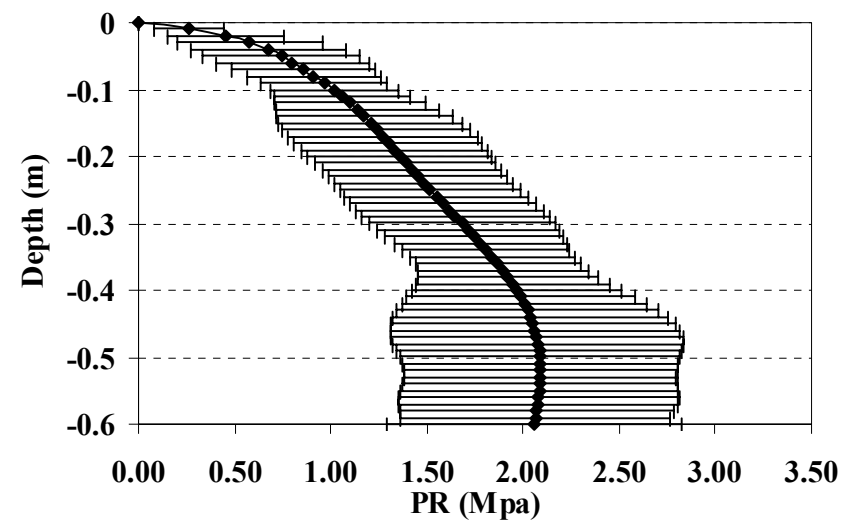

Fig. 2. Mean and standard deviation of 50 depth-dependent PR profiles recorded over the 6.5 ha experimental field.

on average had a similar sand percent (Table 1), and soil water content was near field capacity conditions, as previously stated; therefore the trend of increasing mean PR values with depth might be mainly related to increasing bulk density. In spite of the relatively moderate $\mathrm{CV}$ values of mean PR over the entire field, for successive depths, both the vertical variability within each PR profile and the between profile variability (Fig. 3) were strong enough to suggest wide vertical and lateral discontinuities in texture and soil water content over the experimental field. Depth-dependent and horizontal PR variability of individual profiles will be next addressed.

Mean PR for each of the 50 profiles measured ranged from 0.817 to $2.396 \mathrm{MPa}$, whereas maximum PR was between 1.193 and $5.201 \mathrm{MPa}$. For 36 out of 50 profiles, PR was higher than $2.0 \mathrm{Mpa}$ in some layer within 0 and $60 \mathrm{~cm}$ depth. There is broad evidence indicating that plant root growth may be limited above the $2.0 \mathrm{Mpa}$ PR thresholds (e.g. Häkansson et al., 1988; Soane and Van Ouwerkerk, 1994). Our PR measurements have been performed with a relatively high water content, near field capacity. Therefore, the coarse-textured soil of the experimental field can be considered as prone to compaction and limitations induced by compaction are expected to increase as the soil water content decreases, during dry periods.

Coefficients of variation for individual PR profiles varied from 24.1 to $74.2 \%$. The skewness of the PR frequency distributions was in the range from -2.673 to 0.862 , which indicates, in general, a lack of symmetry. Most of the PR data sets (with 4 exceptions in 60 datasets) were negatively skewed. Negative skewness is associated to a long left tail, which indicates relatively few low values and that the bulk of the values are on the right of the mean. Kurtosis ranged from -1.591 to 7.229 , with a similar number of profiles (i.e. 25) with positive and negative values; three of the profiles exhibit a very flat histogram, matching values for kurtosis higher than 2.5.

Different patterns of depth-dependent PR profiles have been recorded, as illustrated in Fig. 3, where three main types can be observed. Thus, Fig. 3a (profiles \#7, \#15, \#29, and

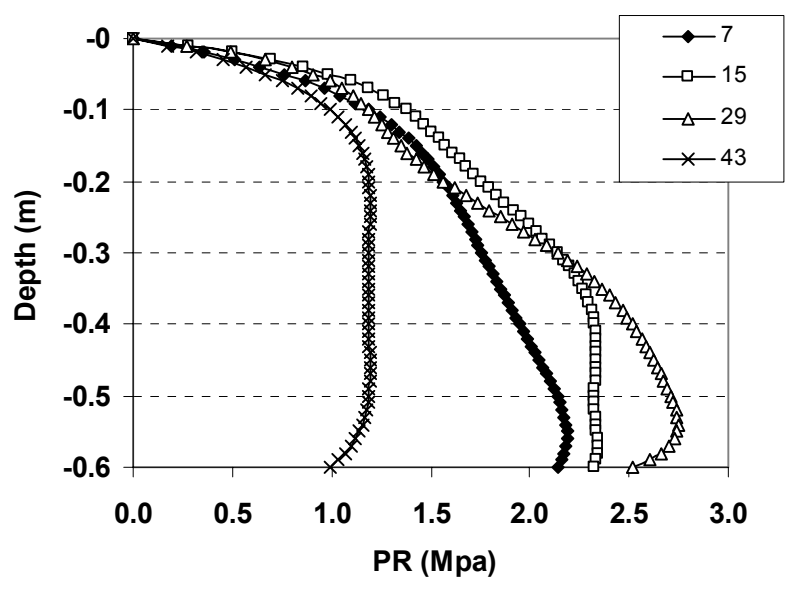

(a) Type A

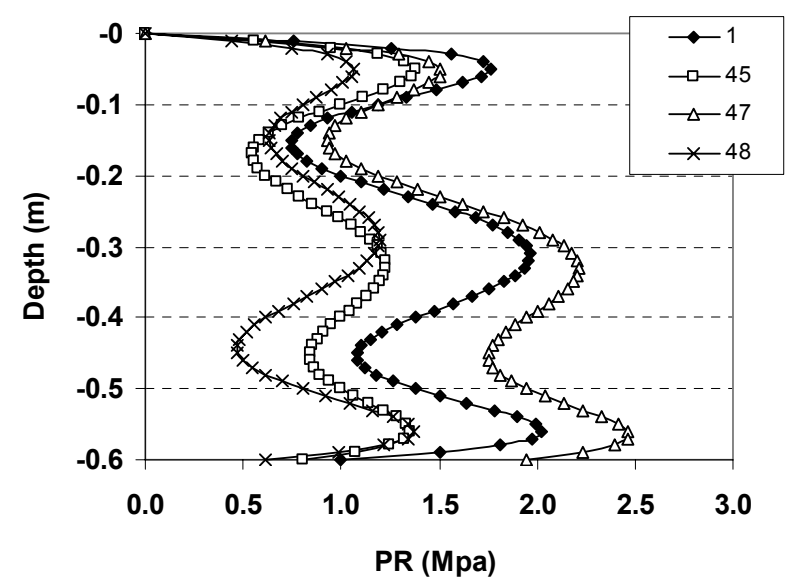

(b) Type B

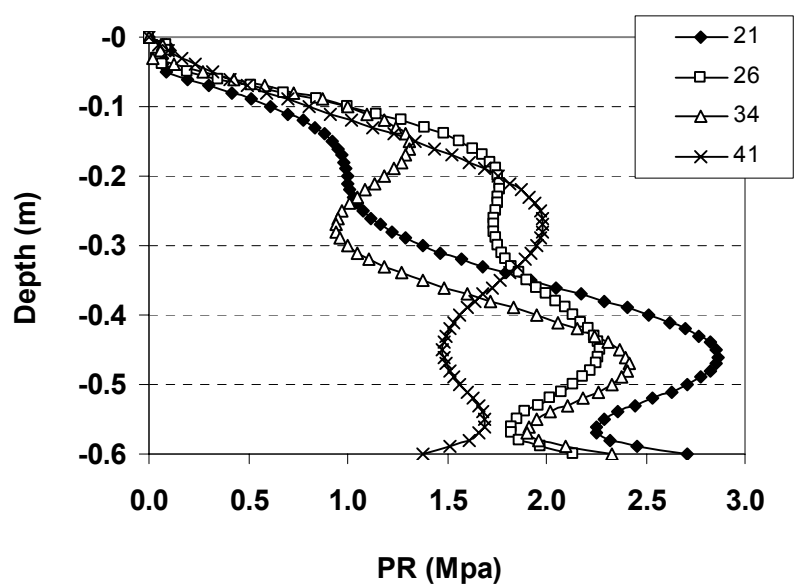

(c) Type $\mathrm{C}$

Fig. 3. Selected types of single depth-dependent PR profiles.

\#43) shows PR gradually increases with depth until a moreor-less constant value is reached; CVs ranged from 24.5 to $37.9 \%$. In contrast, Fig. 3b (profiles \#1, \#45, \#47, and \#48) illustrates a pattern of PR variation in depth, with successive 
"ridges" and "plains"; three maxima and three minima can be recognized at the top, middle and bottom of the profile, and in this sense this depth-dependent pattern is multimodal. CVs of profiles in Fig. $3 \mathrm{~b}$ were very much alike, varying from 30.7 to $31.8 \%$. Profiles in Fig. 3c (\#21, \#26, \#34, and \#41) appear to be less ordered, showing increasing PR with increased depth near the soil surface, and then one or two "ridges" irregularly placed at greater depths so that they do not exhibit a common pattern of depth-dependent PR variation; accordingly, these profiles were characterized by CVs higher than $40 \%$.

\subsection{Multifractal analysis of depth-dependent soil penetration resistance}

Plots of the normalized measure $\chi(q, \delta)$ versus the measurement scale, $\delta$, were examined, for all the statistical moments of interest, $q$, to find out whether or not depth-dependent PR obeyed power law scaling. All the partition functions were constructed for successive box sizes in steps of $2^{k}, k=0$ to $k=5$. For all statistical moments, $q$, in the range [ -8 , 8], the logarithm of $\chi(q, \delta)$, versus the logarithm of $\delta$ fitted a linear model $\left(r^{2}>0.977\right)$ in the imposed range of box sizes, $0<k<5$. Figure 4 shows two selected $\log$-log plots of $\chi(q, \delta)$ versus $\delta$, which correspond to the worst (profile \#34) and the best (profile \#45) linear fits obtained for the $50 \mathrm{PR}$ profiles studied.

Next, singularity spectrum and generalized dimension spectrum were estimated using Eqs. (2) and (3) listed in Table 2 , respectively. In both cases parameter $q$ was chosen in the interval $[-8,8]$, and calculations were performed in increments of 0.5 . To obtain the singularity spectrum, a minimum value of $R^{2}=0.90$ for coefficients of determination of the straight lines relating numerator versus denominator in Eq. (2) was taken up; thus, pairs of values $f(\alpha)$ versus $\alpha$ below this threshold were not accepted in the $f(\alpha)-\alpha$ plots. Following this rule, all the positive moments in the interval $\Delta q_{+}=[0,8]$ were used to construct the $f(\alpha)-\alpha$ plots of the $50 \mathrm{PR}$ profiles, whereas the interval of negative moments in these plots varied from $\Delta q_{+}=[0,-2.5]$ to $\Delta q_{+}=$ $[0,-8]$. Selected examples of singularity spectra are shown in Fig. 5, matching the PR patterns previously depicted in Fig. 3. These spectra were concave down parabolic curves with an asymmetrically long right part for all the studied PR depth-dependent profiles, but there were various degrees of asymmetry. The Hölder exponents of order zero, $\alpha_{0}$, i.e. the scaling exponent corresponding to the maximum $f(\alpha)$, varied between 1.020 and 1.112; determination coefficients in estimating $\alpha_{0}$ were $R^{2} \geq 0.998$. Parameters $\alpha_{\max }$ and $\alpha_{\min }$ were calculated with determination coefficients $R^{2}>0.957$ and $R^{2}>0.979$, respectively. It is worth remembering that in a homogeneous fractal system the singularity spectrum would be reduced to a single point; therefore, shape, asymmetry and goodness of fit statistics of the $f(\alpha)-\alpha$ curves obtained support the hypothesis of the singular behaviour of PR profiles.

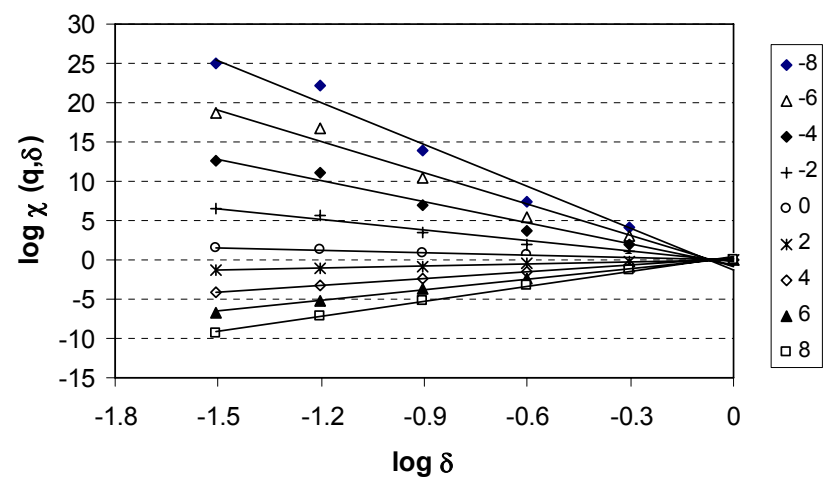

(a) Profile \# 34

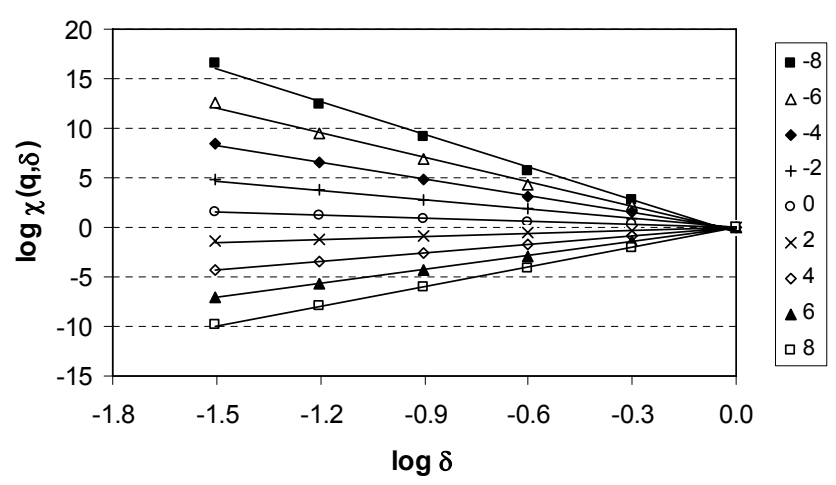

(b) Profile \# 45

Fig. 4. Examples of log-log plots of the partition function, $\chi(q, \delta)$, versus depthdependent resolution, $\delta$. Plots with the worst (profile \#34) and the best (profile \#45) linear correlations are shown.

The width or amplitude of the singularity spectrum, denoted either as $\left(\alpha_{q-}-\alpha_{q+}\right)$ or as $\left(\alpha_{\max }-\alpha_{\min }\right)$, is an indicator of multifractality because it provides information on the diversity of he scaling exponents of the measure. Thus, the wider the $f(\alpha)-\alpha$ spectrum, the higher the heterogeneity in the scaling indices and in the distribution of the PR profiles. The narrowest spectra (Fig. 5b), indicating a lower scaling heterogeneity or multifractality of the measure, were found for PR profiles typified by three successive "ridges" more or less regularly spaced from top to bottom depicted in Fig. 3b. The PR profile types depicted in Fig. 3a and c were translated into wider distributions of the singularity spectra, shown in Fig. 5a and b, respectively, which are indicative of a higher inhomogeneity or multifractality. These results are compatible with a high regularity in the intermittent high and low values of the PR profiles in Fig. 3b, which contrast with Fig. $3 c$ with more changes and unevenness in the successive PR maxima and minima values.

Shape and asymmetry of the $f(\alpha)-\alpha$ curves may also provide much information about the inner structure of the measure and can also be employed to assess its heterogeneity. For example, symmetry of the $\alpha-f(\alpha)$ plots has been frequently 


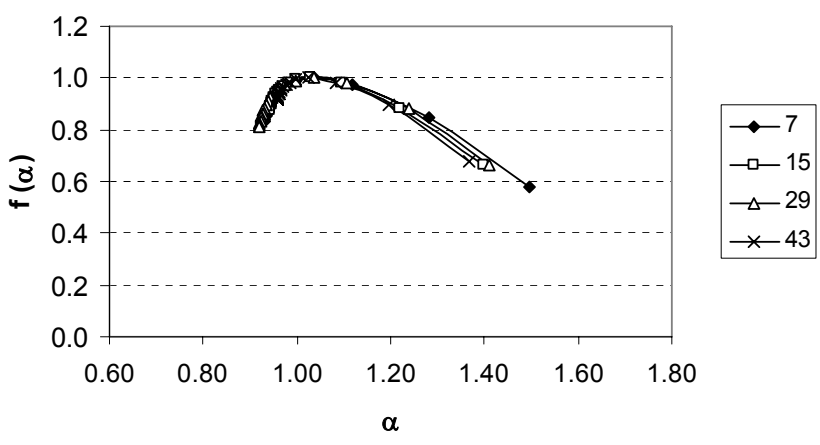

(a) Type A

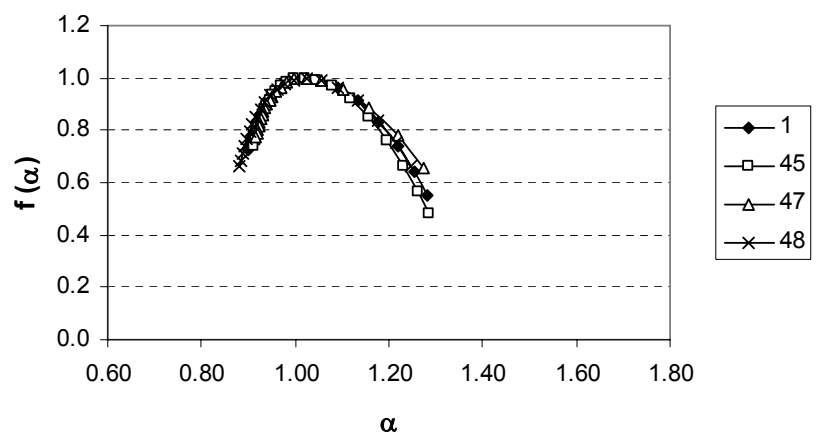

(b) Type B

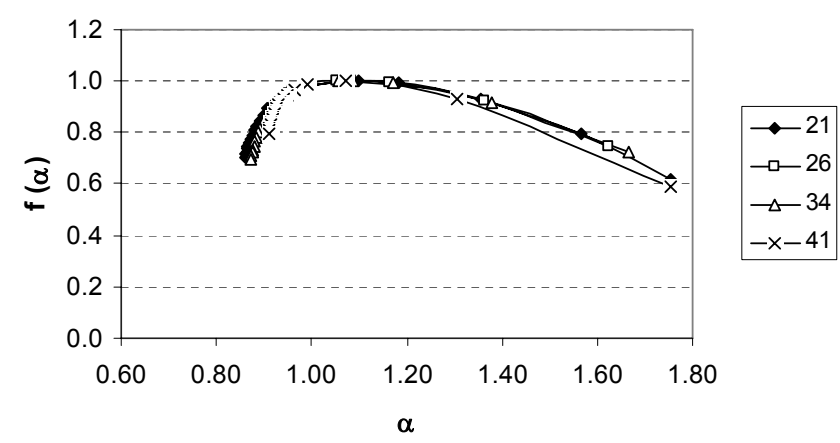

(c) Type C

Fig. 5. Selected examples of singularity spectra (types as in Fig. 4).

quantified using several indices relying on the widths of the respective right $(\mathrm{R})$ and the left $(\mathrm{L})$ branches, such as the ratio $\mathrm{L} / \mathrm{R}=\left(\alpha_{0}-\alpha_{\min }\right) /\left(\alpha_{\max }-\alpha_{0}\right)$ in Macek and Wawrzaszek (2009) or the difference $\mathrm{R}-\mathrm{L}=\left(\left(\alpha_{\max }-\alpha_{0}\right)-\left(\alpha_{0}-\alpha_{\min }\right)\right)$ in San José Martínez et al. (2010). Also, the presence of extremely high and extremely low data values and dominance of either low or high data are related to the left $(q \gg 1)$ and right $(q \ll 1)$ parts of the $f(\alpha)$ spectrum, respectively. Overall, the right branch of the singularity spectrum, $R$, was wider than the left branch, $\mathrm{L}$, and accordingly the ratio $\mathrm{L} / \mathrm{R}$ was below the unity $(\mathrm{L} / \mathrm{R}<1)$. Asymmetry towards the right indicates dominance of the highest singularity exponents, $\alpha$, which correspond to low concentrations of the measure.
Therefore, in terms of the studied variable, the right skewed spectrum is the result of a higher scaling heterogeneity in the distribution of lower PR data values. Again, the relative degree of asymmetry was lower for PR profiles described by a multimodal pattern of variation in depth. Notice also that the general shape of the singularity spectra in Fig. 5 is compatible with the negative skewness of most of the corresponding experimental PR profiles.

On the other hand, low $f(\alpha)$ values correspond to rare events, whereas the highest value of $f(\alpha)$ matches the capacity dimension. In general, the right-hand side $(q<0)$ of the $\alpha-f(\alpha)$ plots was also longer than the left-hand side $(q>0)$, revealing that the geometrical size of points with the largest exponents, $\alpha$, was smaller. Therefore, the narrower and shorter left-hand side of the singularity spectrum suggest dominance of highest PR values and that these were quite similar to each other, as compared to the lowest PR values that were less frequent and showed more differences between one another along the profile.

Next PR singularity spectra will be typified by parameter $\left(\alpha_{0}-\alpha_{\min }\right)=\left(\alpha_{0}-\alpha_{8}\right)$ as well as the Hölder exponent of order zero, $\alpha_{0}$. This is because $\alpha_{\max }$ values of all the $f(\alpha)-\alpha$ plots were computed for a similar statistical moment $\left(\Delta q_{+}=8.0\right)$ and had smaller statistical errors, while $\alpha_{\min }$ values correspond to diverse moments $\left(-8 \leq \Delta q_{-} \leq\right.$ $-2.5)$ and showed larger statistical errors. Large statistical errors for moments $q \ll 0$ are known to be prevailing in multifractal analysis (e.g. Macek and Wawrzasek, 2009; PazFerreiro et al., 2010).

Generalized dimension, $D_{q}$, was estimated in the interval of $q$ moments $\Delta q=[-8,8]$ with Eq. (3b), except for $q=1$, where Eq. (3c) was used. As expected, coefficients of determination were highest for $q=0\left(R^{2}=1.00\right)$ and decreased with increased $|q|$ for the $50 \mathrm{PR}$ profiles studied. Thus, the values of $R^{2}$ were higher than $0.998,0.994,0.984$ and 0.962 for $q=1, q=2, q=8$ and $q=-8$, respectively. Examples of Rényi dimension spectra, $D_{q}$, calculated for $0.5 q$ steps in the range of moments from $q=-8$ to $q=8$ together with their standard error bars are shown in Fig. 6. Rènyi spectra follow a typical monotonically decreasing trend with increasing $q$ values, which can be described by a sigma-shaped curve. The $D_{q}$ function crosses through 1.0 at $q=0$ and approaches minimum and maximum values as $q \gg 1$ and $q \ll-1$, respectively. The absolute differences $\left(D_{0}-D_{q}\right)$ notably increase as the absolute value of $q$ grows in the two branches of the sigma-shaped curves. On the other hand, the curvature of the $D_{q}$ functions was always much higher for negative than for positive values of $q$, which is in accordance with the fact that the singularity spectra had a wider right branch that corresponds to the smallest concentrations of the measure.

The difference $\Delta D_{q}=\left(D_{-8}-D_{8}\right)$ between the most negative $(q=-8)$ and the most positive $(q=8)$ moments has been also employed as a measure of heterogeneity (e.g. Caniego et al., 2006; Paz-Ferreiro et al., 2010). Various 


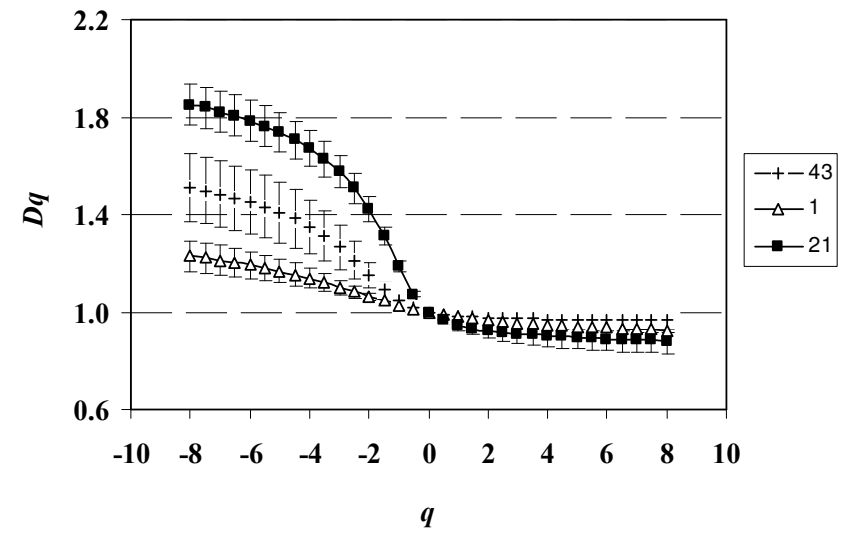

Fig. 6. Selected examples of generalized dimension spectra.

degrees of heterogeneity are shown in the selected Rényi spectra (Fig. 6), which correspond to CV values of $24.5 \%$ (profile\# 43), $30.7 \%$ (profile \#1) and $60.0 \%$ (profile \#21). It is worth noting, however, that the width of the generalized dimension, $\left(D_{-8}-D_{8}\right)$, had a different rank than $\mathrm{CV}$. This is because multifractal parameters reflect major aspects of the spatial organization hidden in the PR data series, while CV is a simple measure of data variability, which does not account for the spatial structure of the points measured along a profile (Everstz and Mandelbrot, 1992).

The capacity dimension, $D_{0}$, was not significantly different from 1 in all of the 50 data series. The entropy or information dimension, $D_{1}$, of PR varied between 0.927 and 0.984. $D_{0}$ and $D_{1}$ would take the same value for a monofractal scaling type. Again the multifractal behaviour of depthdependent PR data series is very clearly expressed in the shape and parameters derived from the generalized dimension, supporting the hypothesis of the singular behaviour of depth-dependent PR data series.

The Hurst exponent, $H$, estimated from the correlation dimension, $D_{2}$, ranged from 0.944 to 0.988 . Thus, $H$ was rather close to 1 and not very different in magnitude from one PR profile to another. The relatively high $H$ figures reflected the presence of strong persistence, i.e. positive autocorrelation, related to long-range dependencies. The strong correlation dependencies with depth for PR profiles are consistent with results reported by San José Martínez et al. (2010) for macroporosity data series. In spite of all the Hurst exponent values obtained being rather close to 1 , this parameter was sensitive to differentiation of main PR patterns of vertical variation; for example, profiles of type A (Fig. 3a) exhibited mean $H$ values significantly lower $(P<0.05)$ than those of profiles of type $\mathrm{B}$ with a multimodal depth-dependent distribution (Fig. 3b), indicative of a relatively smaller autocorrelation of the former.

Indeed, several of the selected multifractal parameters showed strong positive or negative correlations between them. For example, the higher the entropy dimension, $D_{1}$, and the Hurst exponent, $H$, the lower the Hölder exponent of order zero, $\alpha_{0}$, and the degree of multifractality given by indices, such as $\left(\alpha_{\max }-\alpha_{\min }\right)$ or $\left(D_{-8}-D_{8}\right)$. The entropy dimension, $D_{1}$, describes the diversity of the measure (e.g. Caniego et al., 2006; Paz-Ferreiro et al., 2010), so low $D_{1}$ values reflect the fact that most of the measure concentrates in a small size domain, whereas high values of $D_{1}$ indicate that the measure is more evenly distributed. The Hurst exponent, $H$, identifies persistence or long-range autocorrelation (e.g. Pérez et al., 2010; San José Martínez et al., 2010), so low $H$ means a weak persistence and vice versa. Great $\alpha_{0}$ values and wide $\left(D_{-8}-D_{8}\right)$ spectra are characteristic of a high heterogeneous measure. Therefore, in terms of soil PR, the lower the entropy dimension, $D_{1}$, the more clustered (i.e. less evenly distributed), the weaker the persistence, the smaller the autocorrelation and the large the heterogeneity and anisotropy of the depth-dependent penetrometer readings.

Scaling heterogeneity or multifractality in soil properties result from the interaction of various underlying soil forming factors and processes. In agricultural soils, the complexity due to natural factors may be modified by man-made factors (e.g. Biswas et al., 2012). Depth-dependent PR profiles vary with soil properties that may show great spatial heterogeneity (Vaz et al., 2011), which, in turn, may be either relatively constant over time (i.e. particle size distribution, soil mineralogy, organic matter content, etc.) or highly dynamic (i.e. water content, bulk density, porosity, etc.). The joint action of various sources of spatial variability, operating at different scales, showed specific effects for PR profiles measured at each point.

Thus, the scaling property is driven by the number, type and spatial extent of factors and processes that control the dynamics of each soil PR profile. It is well established that PR mostly depend on soil texture, bulk density and water content (e.g. Dexter et al., 2007; Vaz et al., 2011). In turn, soil water content near the soil surface is highly variable (Biswas et al., 2012) because of the influence of external factors such as rainfall and evaporation. Therefore, soil PR may be also indirectly influenced by environmental factors, and soil surface layers are more responsive to these factors than deep soil layers. Bulk density at the surface soil layers is also strongly influenced by tillage operations. Therefore, due to the dynamics of soil water content and soil structure, strength properties near the soil surface are highly variable in nature, which will be also reflected in the scaling property of depth-dependent PR profiles. To summarize, scale heterogeneity in the vertical distribution of soil PR could be driven by various local and non local agents or sources of variation, including soil texture, organic matter content, bulk density, water content and water dynamics, rainfall, evaporation and tillage operations.

The multifractality observed in the PR profiles can be viewed as the result of differences in autocorrelation for the smallest and large fluctuations of the experimental data series. At our experimental scale, PR peaks are most likely 

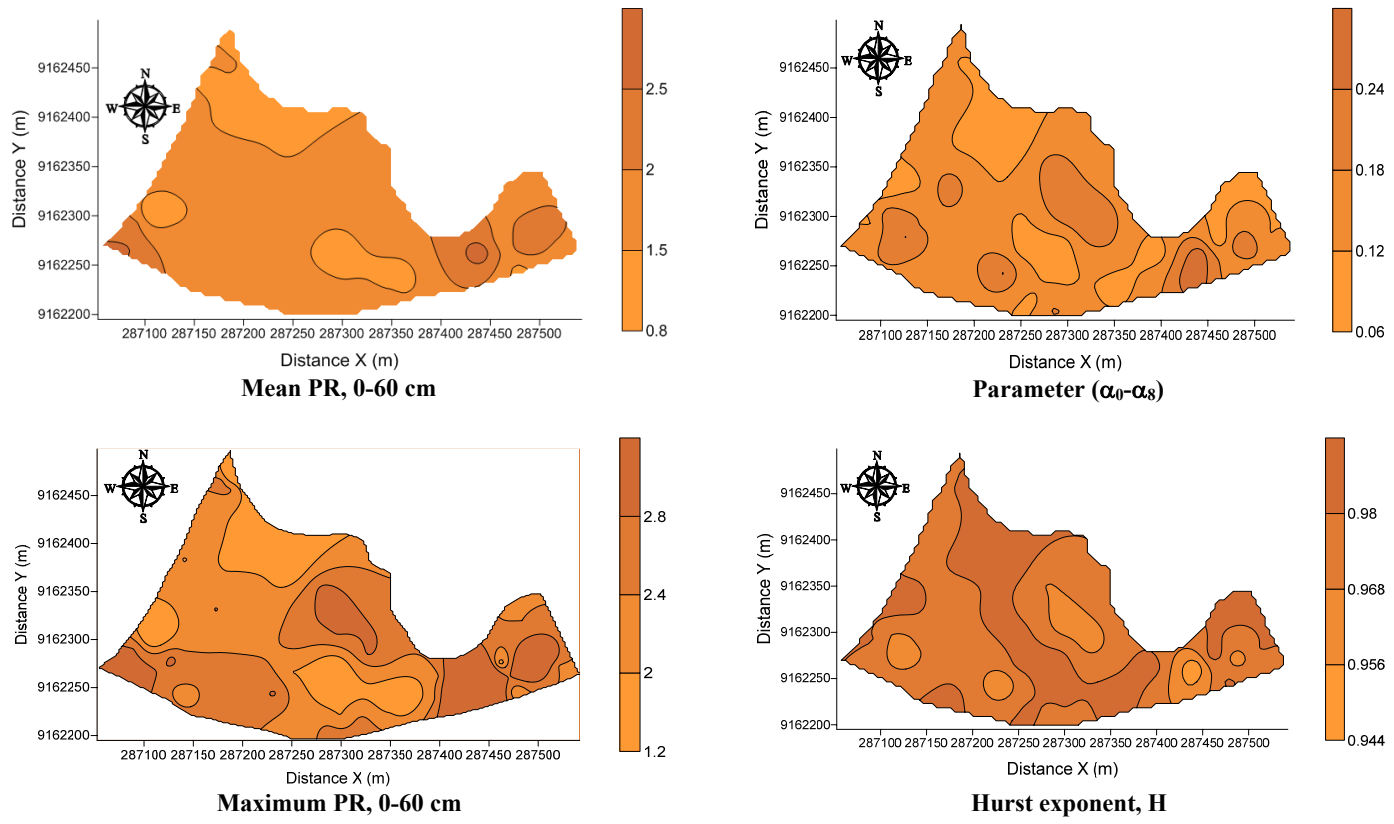

Fig. 7. Maps at the field scale of maximum and mean PR, parameter $\left(\alpha_{0}-\alpha_{8}\right)$, and Hurst exponent, $H$.

associated to the physical obstruction found by the steel cone to enter or to push aside small soil structural units, whereas drops in PR should correspond to voids or layers with loose density. The higher the packing density of a soil layer, the greater the resistance encountered by the penetrometer tip, which results in increasing maximum and mean PR. Moreover, soil texture and soil water content along a vertical PR profile may be far from homogeneous. The above-mentioned factors operate and interact at closely related scales to give rise to the observed PR features. The different PR types analysed showed in general a strong multifractality, although there were differences in the degree of multifractality and also in the degree of symmetry. Since multifractal structures are the outcome of chaotic phenomena, our results may be interpreted as a positive test on the suitability of nonlinear dynamics to analyse PR profiles.

\subsection{Linear and spatial relationship between multifractal and statistical parameters}

Table 3 shows Pearson product moment correlation between selected parameters derived from multifractal analysis, i.e. $D_{-8}, D_{8},\left(D_{-8}, D_{8}\right), D_{1}, H,\left(\alpha_{0}-\alpha_{8}\right)$ and $\alpha_{0}$, and various commonly used PR statistical indices (maximum, mean, standard deviation, CV, skewness and kurtosis). The Hurst exponent, $H$, and $\mathrm{CV}$ showed a negative dependence with the highest correlation coefficient $(r=-0.978)$. $H$ was also significantly and negatively correlated to standard deviation $(r=-0.858)$, maximum PR $(r=-0.733)$, skewness $(r=-0.729)$ and mean PR $(r=0.316)$, but showed a positive correlation to kurtosis $(r=0.557)$. The entropy dimen- sion, $D_{1}$, was also correlated to these descriptive statistical indices, but coefficients of correlation were somewhat lower. Parameter $D_{8}$, cropped from the right branch of $D_{q}$ spectra, showed the highest negative relationship with CV $(r=-0.891)$ and it is worth nothing that $D_{8}$ was even better correlated to skewness $(r=-0.851)$ and kurtosis $(r=$ 0.612 ) than the Hurst exponent, $H$. However, $D-8$, derived from the left branch of $D_{q}$ spectra showed either no correlation or low positive correlations with statistical indices ( $r=0.321$ to standard deviation and $r=0.319$ to CV). Parameter $\left(\alpha_{0}-\alpha_{8}\right)$, in contrast, was positively correlated to $\mathrm{CV}$ $(r=0.947)$, standard deviation $(r=0.782)$, skewness $(r=$ $0.761)$ and maximum PR $(r=0.680)$, exhibiting a negative correlation to kurtosis $(r=-0.620)$. The above-mentioned statistical indices were also significantly correlated to the Hölder exponent of order zero, $\alpha_{0}$, but the sign of the correlation was opposite to that of $H$ and $D_{1}$.

In summary, parameters gathered from the central part of the generalized dimension (i.e. $D_{1}, H$ ) or the singularity spectrum (i.e. the Hölder exponent of order zero, $\alpha_{0}$ ), and those obtained for positive moments of these curves, $q \gg 1$, (i.e. $D_{8}, \alpha_{8}$ and $\left.\left(\alpha_{0}-\alpha_{8}\right)\right)$ showed strong correlations to statistical indices. In contrast, parameters obtained for negative moments, $q \ll 1$, i.e., $D_{-8}, \alpha_{-8}$, showed no or less significant correlation to statistical indices.

The strong correlation between statistical indices and multifractal parameters for $q \gg 1$, such as $D_{8}$ or $\left(\alpha_{0}-\alpha_{8}\right)$, can be attributed to the fact that these parameters represent high or extremely high values of PR that are amplified by positive $q$ values. Again, this is also consistent with the negatively skewed frequency distributions for most of the 
Table 3. Correlation between selected statistical and multifractal parameters.

\begin{tabular}{lcccccc}
\hline & $D_{-8}$ & $D_{8}$ & $D_{1}$ & $H$ & $\alpha_{0}-\alpha_{8}$ & $\alpha_{0}$ \\
\hline Maximum & $\mathrm{ns}$ & $-0.720^{*}$ & $-0.684^{*}$ & $-0.733^{*}$ & $0.680^{*}$ & $0.516^{*}$ \\
Mean & $\mathrm{ns}$ & $\mathrm{ns}$ & $-0.310^{*}$ & $-0.316^{*}$ & $\mathrm{~ns}$ & $0.290^{* *}$ \\
Std & $0.321^{* *}$ & $-0.751^{*}$ & $-0.779^{*}$ & $-0.858^{*}$ & $0.782^{*}$ & $0.699^{*}$ \\
CV & $0.319^{* *}$ & $-0.891^{*}$ & $-0.968^{*}$ & $-0.978^{*}$ & $0.947^{*}$ & $0.811^{*}$ \\
Skewness & $\mathrm{ns}$ & $-0.851^{*}$ & $-0.620^{*}$ & $-0.729^{*}$ & $0.761^{*}$ & $0.369^{* *}$ \\
Kurtosis & $\mathrm{ns}$ & $0.612^{*}$ & $0.557^{*}$ & $0.596^{*}$ & $-0.620^{*}$ & $-0.423^{* *}$ \\
\hline
\end{tabular}

* denotes $P>0.01$, ** denotes $P>0.05$, and ns stands for not significant.

std is standard deviation; $\mathrm{CV}$, coefficient of variation; $D_{-8}, D_{8}$ and $D_{1}$, generalized dimension for

moments $q=-8,8$ and 1 , respectively; $H$, Hurst exponent; and $\alpha_{8}$ and $\alpha_{0}$, singularity strength for $q=8$ and 0 , respectively.

depth-dependent PR profiles, with the bulk of its values on the right of the mean, and a few small values originating from the relatively long left tail. Moreover, PR profiles with positive skewness exhibited the lowest values of $\left(\alpha_{0}-\alpha_{8}\right)$, which also is in agreement with the longer right tail of its corresponding frequency distribution.

Maximum PR values were also negatively correlated to parameters of the central part of the $D_{q}$ spectra such as $D_{1}$ and $H$, suggesting that layers with the highest strength are associated to decreasing evenness and persistence of the data sets. A few layers with high PR values increase mean PR and its corresponding $\mathrm{CV}$, and therefore the negative correlation between these statistical parameters to $D_{1}$ and $H$ is also an expected result.

Until now, multifractal analysis had not been used to describe the depth-dependent patterns of penetrometer measures. Multifractal theory takes advantage of the independence of multifractal parameters over a range of scales and the assumption of a non-specific distribution of the measure. Assuming a non-specific distribution of PR allows for characterization of features such as heterogeneity of high and low values of soil resistance and the presence of rare events over the measured depth.

Subsequently, multifractal analysis provides insight relating to the higher positive and negative statistical moments, $q$, and how the higher moments change with scale. Hence, not only parameters associated to high PR values (i.e. $D_{8}$ and $\left(\alpha_{0}-\alpha_{8}\right)$ ), but also those related low PR values (i.e. $D_{-8}$ or $\left.\left(\alpha_{-8}-\alpha_{0}\right)\right)$ are required to fully characterize the depthdependent behaviour of data sets taken by penetrometer. In other words, description of soil PR relies not only on the distribution of the highest resistance values but also on the distribution of the lowest ones (e.g. Vaz et al., 2011).

It follows that multifractal parameters, based on the global statistical properties of the depth-dependent data series, reflect many of the major aspects of the vertical arrangement of PR readings, while statistical parameters are basic measures that do not account for a thorough description of such aspects. As an example, in Fig. 6, the $D_{8}$ value of profile \#43 is smaller than that of profile \#1. However, examination of the left part of the graph shows that the $D_{8}$ value (associated to the impact of low penetration resistance data) was much more important for profile \#43 than for profile \#1.

Summarizing, multifractal analysis provides a powerful method to amplify differences between depth-dependent PR profiles as well as to distinguish themselves in more detail. Clearly, this study shows that multifractal methods go beyond statistical methods, allowing a more consistent and better suited description of the inner structure of PR depthdependent profiles that complete the information provided by descriptive statistics.

Figure 7 shows the spatial distribution maps for mean and maximum PR at the $0-60 \mathrm{~cm}$ depth, as well as the Hurst exponent, $H$, and parameter $\left(\alpha_{0}-\alpha_{8}\right)$, constructed by the inverse distance method. In spite of the relatively high water content at the date of measurement several small patches of the experimental field exhibited mean PR resistance higher than the 2.0 MPa threshold. Accordingly, maximum PR maps showed that in most of the field surface, PR values higher than 2.0 MPa occurred at least in one layer within the 0 $60 \mathrm{~cm}$ depth.

At the field scale, in general, areas with high mean or maximum PR values correspond to areas with low $H$ values. Therefore, areas with highest levels or with highest risk of soil compaction are expected to exhibit PR profiles relatively less persistent, thus with a lower autocorrelation and less evenly distributed than areas with lowest risk of soil compaction. Likewise, areas with high mean or maximum PR values, associated to compaction problems, correspond to areas with the highest $\left(\alpha_{0}-\alpha_{8}\right)$ values resulting from more heterogeneity in the distribution of the higher PR data values.

The above results also suggest that adequate assessment of PR variability would need a greater sampling intensity in areas exhibiting a relatively weak depth-dependent persistence or autocorrelation and vice versa. In other words, this means higher sampling intensity is required in areas with the highest scaling heterogeneity.

The location of areas with PR levels that can be limiting for root development and plant growth is practical information of interest for safe soil and crop management, 
particularly in situations that require alleviation of soil compaction. The combination of spatial variability survey and multifractal analysis appears to be useful to manage soil compaction. As previously stated, in our experimental conditions, PR values were relatively low because of the high water content. Further research is needed to test the options open here for PR data series recorded in different soil types and management conditions, and also to assess the spatial variability of $\mathrm{PR}$ in our soil for drier soil conditions.

\section{Conclusions}

We studied 50 depth-dependent soil resistance profiles measured by penetrometer at the field scale on a sandy soil under high moisture conditions. Vertical PR profiles behave like a multifractal system and its multifractal structure was represented by the singularity spectrum, $\alpha$, versus $f(\alpha)$, and the generalized dimension, $D_{q}$. Various degrees of multifractality (scaling heterogeneity) were present in our data series and the highest multifractality corresponded to vertical PR profiles described by an irregular multimodal pattern of variation in depth.

Overall, singularity spectra were asymmetrical and shifted to the right, which is compatible with a greater heterogeneity of the low values in all the PR data sets. Again, the relative degree of asymmetry was lower for depth-dependent PR profiles exhibiting a multimodal pattern of variation. These results are also consistent with the negative skewness coefficient of most of the studied data sets.

The Hurst exponent, $H$, varied between 0.944 and 0.988 , indicating the presence of a rather strong persistence or long-range dependence. Autocorrelation or long-range dependence was higher for profiles with a multimodal pattern of PR distribution.

Multifractal analysis completed descriptive statistics, yielding information about changes with scale of the higher moments, which gives a deep insight into the inner structure of PR depth-dependent profiles. Therefore, multifractal analysis provided a powerful method to amplify differences between depth-dependent PR profiles and contributed to distinguish themselves in more detail. In addition, combination of spatial variability survey and multifractal analysis appear to be an useful tool to manage soil compaction.

Acknowledgements. The authors would like to thank the federal organization CNPq and the Pernambuco State organization FACEPE for the financial support and awarded fellowship that enabled this work to be done. J. Paz-Ferreiro was supported by a Juan de la Cierva contract.

Edited by: S. Gaci

Reviewed by: R. G. Moreno and two anonymous referees

\section{References}

Armstrong, A. C.: On the fractal dimensions of some transient soil properties, J. Soil Sci., 37, 641-652, 1986.

ASAE: Soil cone penetrometer, ASAE standards, American Soc. Agric. Eng., S313, 2, St. Joseph, MI, 466 pp., 1986.

Biswas, A., Zeleke, T. B., and Si, B. C.: Multifractal detrended fluctuation analysis in examining scaling properties of the spatial patterns of soil water storage, Nonlin. Processes Geophys., 19, 227-238, doi:10.5194/npg-19-227-2012, 2012.

Brasil, Ministry of Agriculture: Levantamento detalhado dos solos da Estação Experimental de Itapirema, Rio de Janeiro, 84 pp., (Boletim Técnico, 12, 85 pp., 1969 (in Portuguese).

Brasil, Ministry of Agriculture: Levantamento exploratórioreconhecimento de solos do Estado de Pernambuco, Recife, Brazil 2vol., DNPEA, Boletim Técnico, 26; SUDENE-DRN, Série Pedologia, 14, 359 pp., 1972 (in Portuguese).

Burrough, P. A.: Multiscale sources of spatial variation in soil: I. The application of fractal concepts to nested levels of soil variation, J. Soil Sci., 34, 577-597, 1983.

Burrough, P. A. and Mc Donnell, R.: Principles of Geographical information systems, Oxford Univ. Press, UK, 346 pp., 1998.

Camargo, O. A., Moniz, A. C., Jorge, J. A., and Valadares, J. M. A. S.: Métodos de análise química, mineralógica e física de solos do Instituto Agronômico de Campinas, Instituto Agronômico, Campinas, Boletim técnico, 106, 94 pp., 1986 (in Portuguese).

Caniego, J., Ibáñez, J. J., and San José Martínez, F.: Selfsimilarity of pedotaxa distributions at the planetary scale: a multifractal approach, Geoderma, 134, 306-317, 2006.

Caridad-Cancela, R., Vidal Vázquez, E., Vieira, S. R., Abreu, C. A., and Paz González, A.: Assessing the spatial uncertainty of mapping trace elements in cultivated fields, Commun. Soil Sci. Plan., 36, 253-274, 2005.

Castrignanó, A., Maiorana, M., Fornaro, F., and López, N.: 3D spatial variability of soil strength and its change over time in a drum wheat field in southern Italy, Soil Till. Res., 65, 95-108, 2002.

Chhabra, A. B. and Jensen, R. V.: Direct determination of the $f(\alpha)$ singularity spectrum, Phys. Rev. Lett., 62, 1327-1330, 1989.

Da Silva, A. P., Kay, D. B., and Perfect, E.: Characterization of the least limit water range of soils, Soil Sci. Soc. Am. J., 58, 17751781, 1994.

Dexter, A. R., Czyz, E. A., and Gate, O. P.: A method for prediction of soil penetration resistance, Soil, Till. Res., 93, 412-419, 2007.

EMBRAPA (Brazilian Agricultural Research Corporation): Brazilian System of Soil Classification, Rio de Janeiro, Brazil, 412 pp., 2006 (in Portuguese).

Everstz, C. J. G. and Mandelbrot, B. B.: Multifractal measures, in: Chaos and Fractals, edited by: Peitgen, H., Jürgens, H., and Saupe, D., Springer, Berlin, 921-953, 1992.

Falconer, K.: Fractal geometry. Mathematical foundations and applications, John Wiley and Sons, Chichester, 1997.

Feder, J.: Fractals, Plenum Press, New York, 283 pp., 1988.

Folorunso, O. A., Puente, C. E., Rolston, D. E., and Pinzón, J. E.: Statistical and fractal evaluation of the spatial characteristics of soil surface strength, Soil Sci. Soc. Am. J., 58, 284-294, 1994.

García Moreno, R., Díaz Álvarez, M. C., Tarquis, A. M., Paz González, A., and Saa Requejo, A.: Shadow analysis of soil surface roughness compared to the chain set method and direct measurement of micro-relief, Biogeosciences, 7, 2477-2487, doi:10.5194/bg-7-2477-2010, 2010. 
Grassberger, P.: Generalized dimensions of strange attractors, Phys. Lett., 97A, 227-230, 1983.

Guèrif, J.: Factors influencing compaction induced stress in soil strength, Soil Till. Res., 16, 167-178, 1990.

Häkansson, I., Voorhees, W. B., and Riley, H.: Vehicle and wheel factors influencing soil compaction and crop response in different traffic regimes, Soil Till. Res., 11, 239-282, 1988.

Halsey, T. C., Jensen, M. H., Kadanoff, L. P., Procaccia, I., and Shraiman, B. I.: Fractal measures and their singularities: The characterization of strange sets, Phys. Rev. A, 33, 1141-1151, 1986.

Hentschel, H. G. E. and Procaccia, I. The infinite number of generalized dimensions of fractals and strange attractors, Physica D, 8, 435-444, 1983.

Huang, C. H. and Bradford, J. M.: Applications of a Laser Scanner to Quantify Soil Microtopography, Soil Sci. Soc. Am. J., 56, 14 21, 1992.

Leland, W. E., Taqqu, M. S., Willinger, W., and Wilson, D. V.: On the self-similar nature of ethernet traffic (extended version), IEEE/ACM Transactions on Networking (TON), 2, 1-15, 1994.

Letey, J.: Relationship between soil physical properties and crop production, Adv. Soil Sci., 1, 277-294, 1985.

Macek, W. M. and Wawrzaszek, A.: Evolution of asymmetric multifractal scaling of solar wind turbulence in the outer heliosphere, J. Geophys. Res., 114, A03108, doi:10.1029/2008JA013795, 2009.

Mandelbrot, B. B. and Van Ness, J.: Fractional Brownian motion, fractional noises and applications, J. SIAM, 10, 422-437, 1968.

Miranda, J. G. V., Montero, E. Alves, M. C., Paz González, A., and Vidal Vázquez, E.: Multifractal characterization of saprolite particle-size distributions after topsoil removal, Geoderma, 134, 373-385, 2006.

O'Sullivan, M. F., Diskon, J. W., and Campell, D. J.: Interpretation and presentation of cone resistance data in tillage and traffic studies, J. Soil Sci., 38, 137-148, 1987.

Pachepsky, Y., Yakovchenko, V., Rabenhorst, M. C., Pooley, C., and Sikora, L. J.: Fractal parameters of pore surfaces as derived from micro micromorphological data: effect of long-term management practices, Geoderma, 74, 305-319, 1996.

Paz, A., Taboada, M. T., and Gómez, M. J.: Spatial variability in topsoil micronutrient contents in a one-hectare cropland plot, Commun. Soil Sci. Plan., 27, 479-503, 1996.

Paz-Ferreiro, J., Miranda, J. G. V., and Vidal Vázquez, E.: Multifractal analysis of soil porosity based on mercury injection and nitrogen adsorption, Vadose Zone J., 9, 325-335, 2010.

Pérez, L., Millán, H., and González-Posada, M.: Spatial complexity of soil plow layer penetrometer resistance as influenced by sugarcane harvesting: A prefractal approach, Soil Till. Res., 110, 77-86, 2010.
Rényi, A.: On a new axiomatic theory of probability, Acta Math. Hung., VI, 285-335, 1955.

Riedi, R. H., Crouse, S., Ribeiro, V. J., and Baraniuk, R. G.: A multifractal wavelet model with application to network traffic, IEEE T. Inform. Theory, 45, 992-1019, 1999.

Roisin, C. J.: A multifractal approach for assessing the structural state of tilled soils, Soil Sci. Soc. Am. J., 71, 15-25, 2007.

San José Martínez, F., Martín, M. A., Caniego, F. J., Tuller, M., Guber, A., Pachepsky, Y., and García-Gutierrez, C.: Multifractal analysis of discretized X-ray CT images for the characterization of soil macropore structures, Geoderma, 156, 32-42, 2010.

SAS Institute: SAS-Statistical Analysis System, Users Guide, SAS Institute, NC, USA, 1999.

Soane, B. D. and Van Ouwerkerk, C.: Compaction in crop production, Elsevier Science Publishers, Amsterdam, 662 pp., 1994.

Soil Survey Staff: Key to soil taxonomy, 11th Edn., Natural Resources Conservation Service, Washington, DC, 338 pp., 2010.

Stolf, R.: Teoria e teste experimental de fórmulas de transformação dos dados de penetrômetro de impacto em resistência do solo, Rev. Bras. Cienc. Solo, 15, 229-235, 1991 (in Portuguese).

Tarquis, A., Giménez, D., Saá, A., Díaz, M. C., and Gascó, J. M.: Scaling and multiscaling of soil pore systems determined by image analysis, in: Scaling Methods in Soil Physics, edited by: Pachepsky, J., Radcliffe, D. E., and Selim, H. M., CRC Press, 434 pp., 2003.

Usowicz, B. and Lipiec, J.: Spatial distribution of soil penetration resistance as affected by soil compaction: the fractal approach, Ecol. Complex., 6, 263-271, 2009.

Vaz, C. M. P., Manieri, J. M., De Maria, I. C., and Tuller, M.: Modelling and correction of soil penetration resistance for varying soil water content, Geoderma, 166, 92-101, 2011.

Vidal Vázquez, E., Vivas Miranda, J. G. V., and Paz González, A.: Characterizing isotropy and heterogeneity of microtopographic soil surfaces using fractal models, Ecol. Model., 182, 337-353, 2005.

Vidal Vázquez, E., Miranda, J. G. V., and Paz González, A.: Describing soil surface microrelief by crossover length and fractal dimension, Nonlin. Processes Geophys., 14, 223-235, doi:10.5194/npg-14-223-2007, 2007.

Vidal Vázquez, E., García Moreno, R., Miranda, J. G. V., Díaz, M. C., Saá Requejo, A., Paz Ferreiro, J., and Tarquis, A. M.: Assessing soil surface roughness decay during simulated rainfall by multifractal analysis, Nonlin. Processes Geophys., 15, 457-468, doi:10.5194/npg-15-457-2008, 2008.

Vieira, S. R., Millete, J., Topp, G. C., and Reynolds, W. D.: Handbook for geoestatistical analysis of variability in soil and climate data, in: Tópicos em Ciência do Solo, edited by: Alvarez, V. V. H., Schaefer, C. E. G. R., Barros, N. F., Mello, J. W. V., and Costa, J. M., Sociedade Brasileira de Ciência do Solo, Viçosa, v.2, 1-4, 2002. 